\title{
Interplay between hard and soft processes in HYDJET++ model
}

\section{E.E. Zabrodin*}

Department of Physics, University of Oslo, Oslo, Norway

Skobeltzyn Institute of Nuclear Physics, Moscow State University, Moscow, Russia

National Research Nuclear University "MEPhI" (Moscow Engineering Physics Institute),

Moscow, Russia

E-mail: eugen.zabrodin@fys.uio.ng

\section{L.V. Bravina}

Department of Physics, University of Oslo, Oslo, Norway

\section{G.Kh. Eyyubova, I.P. Lokhtin, L.V. Malinina, S.V. Petrushanko, A.M. Snigirev}

Skobeltzyn Institute of Nuclear Physics, Moscow State University, Moscow, Russia

\begin{abstract}
The Monte Carlo event generator HYDJET++ (HYDrodynamics with JETs) contains the description of both soft and hard processes in relativistic heavy-ion collisions by combining the parametrized hydrodynamics with the treatment of jets. The interplay of hard and soft processes describes the violation of the mass hierarchy of meson and baryon elliptic $\left(v_{2}\right)$ and triangular $\left(v_{3}\right)$ flows at intermediate transverse momenta, the fall-off of the flow harmonics after the certain $p_{T}$ threshold, and the worsening of the number-of-constituent-quark (NCQ) scaling of $v_{2}$ and $v_{3}$ at LHC energies compared to that at RHIC ones. The role of this interplay in di-hadron correlations and in production of open and hidden charm in heavy-ion collisions at RHIC and LHC energies is also discussed.
\end{abstract}

High-pT Physics at RHIC and LHC era

October 2 - 52017

Bergen, Norway

\footnotetext{
* Speaker.
} 


\section{Introduction}

HYDJET++ [1] is one of the models included in the event generator pool of CMS Collaboration. It combines the treatment of soft processes with the description of jets propagating in hot and dense partonic medium. The simulation of both soft and hard processes proceeds almost independently. The soft processes are generated by the FASTMC model [2, 3] which employs the parametrized hydrodynamics extended to non-central nuclear collisions to provide elliptic, $v_{2}$, triangular, $v_{3}$, and higher harmonics, $v_{n}, n>3$, of the anisotropic flow. Recall, that the flow harmonics are the coefficients of the Fourier series expansion of azimuthal particle distribution in the transverse plane,

$$
v_{n}=\left\langle\cos \left[n\left(\phi-\Psi_{n}\right)\right]\right\rangle
$$

Here $\phi$ is the particle azimuthal angle, and $\Psi_{n}$ is the azimuth of the event plane of $n$-th harmonic, respectively. Avaraging in Eq.1.1. is performed over all hadrons in an event and over all events.

At any given impact parameter $b$ the program calculates first the number of participating nucleons which permits one to estimate the effective volume $V_{e f f}$ of the overlapping zone. Then, the fireball expansion is governed by Bjorken hydrodynamics. When the temperature drops below a certain threshold value, which is a free parameter of the model, the chemical freeze-out of the system takes place. The fireball continues to expand further until the stage of thermal freeze-out. HYDJET++ is flexible in a sense that it allows also for a scheme with simultaneous chemical and thermal freeze-out. However, the best agreement with the experimental data is obtained in the scenario with two separated freeze-outs. Final-state interactions are represented by two- and threebody decays of resonances. The table of particles and resonances in HYDJET++ is very rich; it contains more than 360 baryonic and mesonic states including the charmed ones.

Hard processes in HYDJET++ are handled by the routine PYQUEN (PYTHIA QUENched) [円]. It takes into account both collisional and radiational energy losses experienced by hard partons in hot and dense medium. The number of hard jets in $\mathrm{A}+\mathrm{A}$ collisions at a given impact parameter is determined by the number of binary nucleon-nucleon $(N N)$ collisions and the integral cross section of the hard $N N$ collision with certain minimum transverse momentum transfer $p_{T}^{m i n}$. Compared to its original version [1], which took into account just elliptic flow [5, 6], the model was further upgraded to (i) better treatment of the hard processes at LHC energies by replacement of standard PYTHIA 6.4 to Pro-Q20 tune [7], (ii) triangular flow [8, 9, 10] and, consequently, higher flow harmonics up to hexagonal flow [11, 12], and (iii) event-by-event fluctuations of the flow harmonics [13]. The anisotropic flow in HYDJET++ arises because of two reasons, namely, spatial (or geometric) anisotropy of the fireball and dynamical (or flow momentum) anisotropy.

In case of spatial elliptic essentricity of the fireball its radius reads

$$
R_{2}(b, \phi)=R_{\mathrm{fr}}\left\{\frac{1-\varepsilon_{2}^{2}(b)}{1+\varepsilon_{2}^{2}(b) \cos \left[2\left(\phi-\Psi_{2}\right)\right]}\right\}^{1 / 2},
$$

where $\Psi_{2}$ is the azimuth of the event plane of elliptic flow and $\varepsilon_{2}^{2}$ is a free parameter of the model. It links the freeze-out radius of the fireball $R_{\mathrm{fr}}(b)$ in a non-central collision to that of a central one $R_{\mathrm{fr}}(b=0) \equiv R_{0}$ as $R_{\mathrm{fr}}(b=0)=R_{0} \sqrt{1-\varepsilon_{2}^{2}(b)}$. To create spatial triangularity, Eq.(1.2) should be 

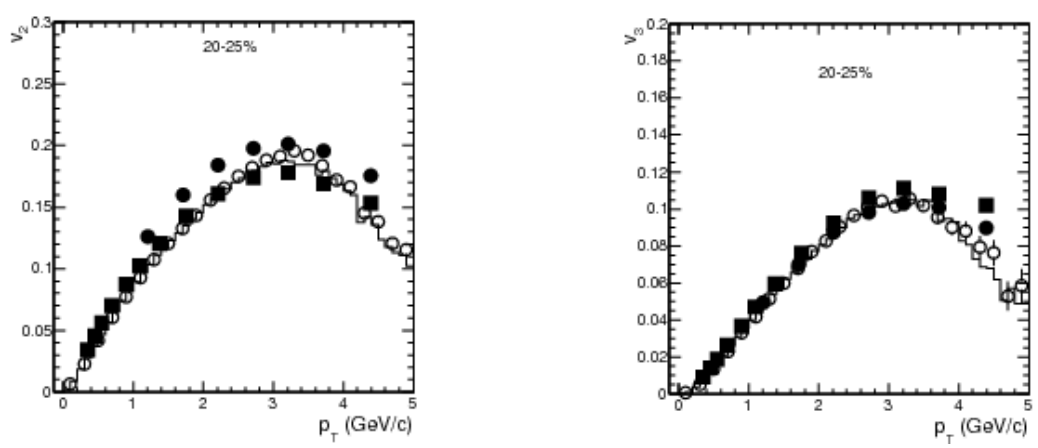

Figure 1: Differential (a) elliptic and (b) triangular flow of charged hadrons in $\mathrm{Pb}+\mathrm{Pb}$ collisions with centrality $20-25 \%$ at $\sqrt{s}=2.76 \mathrm{TeV}$. Solid symbols are data from [15], open circles and hystogram are HYDJET++ calculations, respectively.

modified further [8]

$$
R_{3}(b, \phi)=R_{2}(b, \phi)\left\{1+\varepsilon_{3}(b) \cos \left[3\left(\phi-\Psi_{3}\right)\right]\right\},
$$

containing the azimuth of the event plane of triangular flow, $\Psi_{3}$, and the new free parameter, $\varepsilon_{3}(b)$, responsible for generation of triangular profile of the freeze-out hypersurface. Experiments show that elliptic and triangular flows are not correlated, therefore, their azimuths are randomly distributed in HYDJET++. Note also, that both $\varepsilon_{2}(b)$ and $\varepsilon_{3}(b)$ are normally distributed at any impact parameter around their mean values to account for initial state fluctuations [13]. Therefore, the spatial anisotropies may arise in collisions with $b=0$ as well.

Another source of the anisotropic flow is the dynamical anisotropy. It originates from non-zero angle between the collective flow velocity of the fluid cell $\phi_{\text {cell }}$ and direction of the radius-vector

$$
\tan \phi_{\text {cell }}=\sqrt{\frac{1-\delta_{2}(b)}{1+\delta_{2}(b)}} \tan \phi,
$$

where $\delta_{2}(b)$ is another free parameter responsible for formation of $v_{2}$. To diminish the number of free parameters to be fitted at all centralities, HYDJET++ employs the relation between the $v_{2}, \varepsilon_{2}$, and $\delta_{2}$ obtained within the hydrodynamic approach in [14]

$$
v_{2}\left(\varepsilon_{2}, \delta_{2}\right) \propto \frac{2\left(\delta_{2}-\varepsilon_{2}\right)}{\left(1-\delta_{2}^{2}\right)\left(1-\varepsilon_{2}^{2}\right)} .
$$

This approximation provides fair results up to centrality $\sigma / \sigma_{\text {geo }} \leq 40-45 \%$. At more peripheral events one has to treat $\delta_{2}(b)$ and $\varepsilon_{2}(b)$ completely independently. The last free parameter responsible for the triangular momentum anisotropy is denoted as $\rho_{3}(b)$. Further details of the model can be found in [1, 7, 13] and references therein.

\section{Interplay of hard and soft processes.}

Influence of the interplay on differential elliptic and triangular flows. To see this influence explicitly, we display in Fig. $\mathbb{1}(\mathrm{a}, \mathrm{b})$ two distributions, $v_{2}^{c h}\left(p_{T}\right)$ and $v_{3}^{c h}\left(p_{T}\right)$, obtained in HYDJET++ 


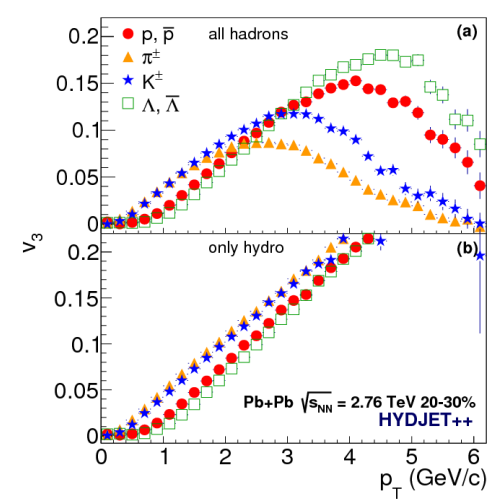

Figure 2: Differential triangular flow of identified hadrons in (a) all processes and (b) only in hydrodynamics in $\mathrm{Pb}+\mathrm{Pb}$ collisions at $2.76 \mathrm{TeV}$ with centrality $20-30 \%$.

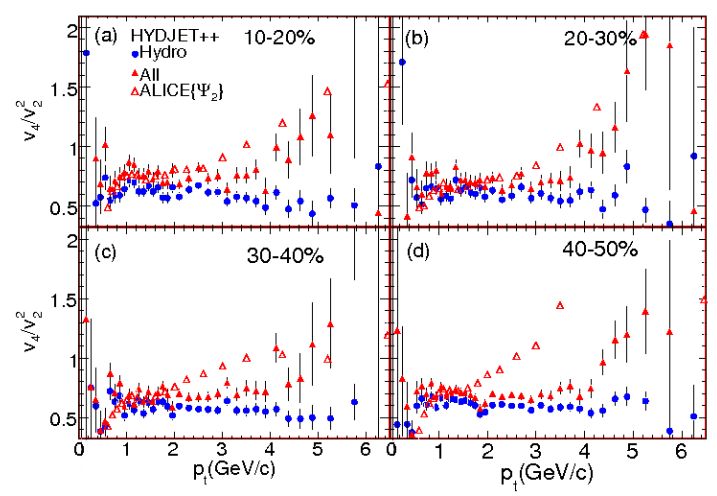

Figure 3: Ratio $v_{4} / v_{2}^{2}$ vs $p_{T}$ for charged hadrons in $\mathrm{Pb}+\mathrm{Pb}$ collisions at $2.76 \mathrm{TeV}$ with different centralities with $(\mathbf{\Delta})$ and without jets $(\bullet)$ compared to ALICE data [17] $(\triangle)$.

calculations of $\mathrm{Pb}+\mathrm{Pb}$ collisions with centrality $20-25 \%$ at $\sqrt{s}=2.76 \mathrm{TeV}$. It is well-known that in ideal hydrodynamics both elliptic and triangular flow increases with rising transverse momentum. The differential flows in Fig. 1. however, drop at $p_{T} \geq 2.5 \mathrm{GeV} / c$. - The collective flow is carried by hadrons produced in soft processes, whereas the flow of hadrons decoupled from jets is essentially zero. Jet particles can develop a weak flow at $p_{T} \geq 3.5 \mathrm{GeV} / c$ because of the jet quenching. Spectrum of hadrons is dominated by particles from the hydro-like processes at $p_{T} \leq 2 \mathrm{GeV} / c$, and by jet particles at $p_{T} \geq 3 \mathrm{GeV} / c$. Therefore, the elliptic or triangular flow of the combined soft+hard hadron spectrum drops after a certain transsverse momentum. For light hadrons the crossing of the soft and hard parts of their $p_{T}$-spectra occurs at smaller transverse momenta compared to those of heavy hadrons. This explains naturally the violation of the mass hierarchy of differential flows (lighter hadrons have larger flow in a low- $p_{T}$ region) and crossing of the meson and baryon flows somewhere at intermediate $p_{T}$. Both features are seen in Fig. 目, which shows the differential triangular flow of identified hadrons for the combined soft+hard spectrum, and only for hadrons coming from hydro processes.

Jets and violation of scaling ratios. It was a hint that the ratios $v_{n}^{1 / n}\left(p_{T}\right) / v_{2}^{1 / 2}\left(p_{T}\right)$ should be almost independent on the transverse momenta. Experimental results seem to confirm this hypothesis [16. In Fig. B we plot the ratio $v_{4} / v_{2}^{2}$ as a function of transverse momentum for four different centralities in $\mathrm{Pb}+\mathrm{Pb}$ collisions at $\sqrt{s}=2.76 \mathrm{TeV}$. The ALICE data show that the ratio increases at $p_{T} \geq 3 \mathrm{GeV} / c$. HYDJET++ calculations with and without jets indicate that the increase emerges because of the contributions from the hard processes.

Jets are also responsible for violation of the number-of-constituent-quark (NCQ) scaling of both $v_{2}$ and $v_{3}$. To observe this scaling one has to divide the flow of a hadronic specie and the transverse kinetic energy $K E_{T}=m_{T}-m_{0}$ by the number of constituent quarks, 2 for mesons and 3

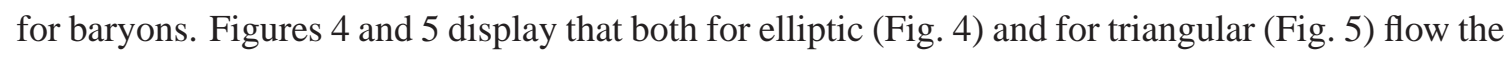
decays of resonances modify the spectra towards the fulfillment of the NCQ scaling. In contrast, because of the jet influence the scaling fulfillment gets worse.

Violation of factorization of dihadron angular harmonics. Here the azimuthal distribution of 


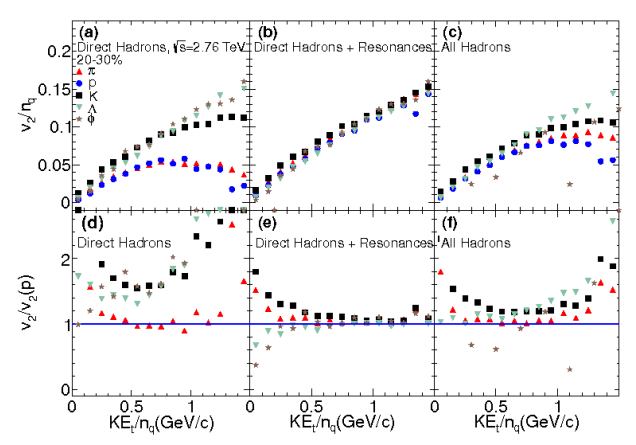

Figure 4: The $K E_{T} / n_{q}$ dependence of the elliptic flow of direct hadrons (left), all hadrons produced only in soft processes (middle), and all hadrons (right) in $\mathrm{Pb}+\mathrm{Pb}$ collisions at $2.76 \mathrm{TeV}$ with centrality $20-30 \%$.

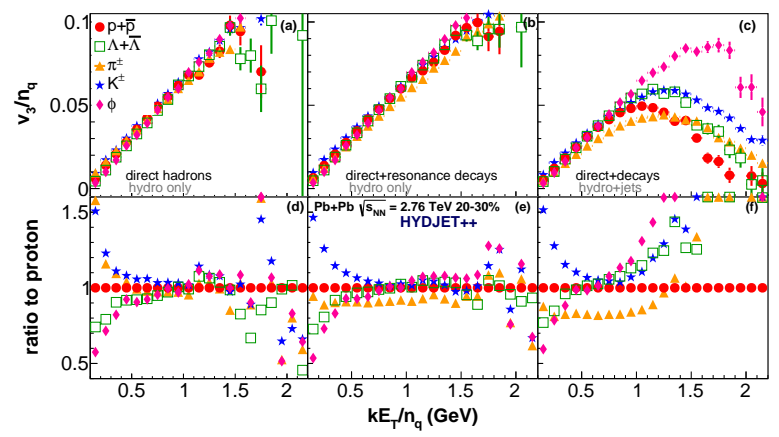

Figure 5: The same as Fig. 4 but for the triangular flow. Here only hadrons directly produced in soft processes are considered in left windows.

two particles, denoted as the "trigger" and the "associated" ones, is expanded in a Fourier series

$$
\frac{d N^{\text {pairs }}}{d \Delta \phi} \propto 1+2 \sum_{n=2}^{\infty} v_{n}\left(p_{T}^{t r}\right) v_{n}\left(p_{T}^{a}\right) \cos (n \Delta \phi)
$$

where $\Delta \phi=\phi^{t r}-\phi^{a}$. In the flow-dominated regime, which takes place at low transverse momenta, the coefficients $V_{n}$ can be presented as

$$
V_{n}\left(p_{T}^{t r}, p_{T}^{a}\right)=v_{n}\left(p_{T}^{t r}\right) \times v_{n}\left(p_{T}^{a}\right)+\delta_{n},
$$

where $v_{n}$ are the corresponding flow harmonics and $\delta_{n}$ is the contribution of non-flow processes. The calculations of $v_{2}, v_{3}$ and $v_{4}$ shown in Fig. 6reveal that factorization $V_{n}=v_{n}^{t r} \times v_{n}^{a}$ works well at $p_{T} \leq 3 \mathrm{GeV} / c$. At higher transverse momenta the non-flow contribution $\delta_{n}$ from the jets dominates, and the factorization is broken (see [18] for details).

Freeze-out of charmed mesons. This problem was studied in [19]. In line with previous investigations, it seems that $J / \psi$-mesons are frozen earlier than the light hadrons. In contrast, for $D$-mesons whose $p_{T}$-spectra are displayed in Fig. 7 the simultaneous thermal freeze-out with other hadrons are not ruled out.

\section{Conclusions}

Interplay of hard and soft processes in relativistic heavy-ion collisions can explain many experimentally observed features, such as (i) drop of the flow harmonics at $p_{T} \geq 3 \mathrm{GeV} / c$; (ii) violation of the mass hierarchy in $v_{2}$ and $v_{3}$; (iii) worsening of the NCQ scaling conditions for both $v_{2}$ and $v_{3}$; (iv) violation of factorization of di-hadron angular harmonics at $p_{T} \geq 3 \mathrm{GeV} / c$. It can also shed light on thermal and non-thermal production of charmed hadrons in these reactions.

Acknowledgements. This work was supported in parts by the Russian Foundation for Basic Research under grant No. 18-02-00155 and the Norwegian Research Council (NFR) under grant No. 255253/F50 CERN Heavy Ion Theory. 

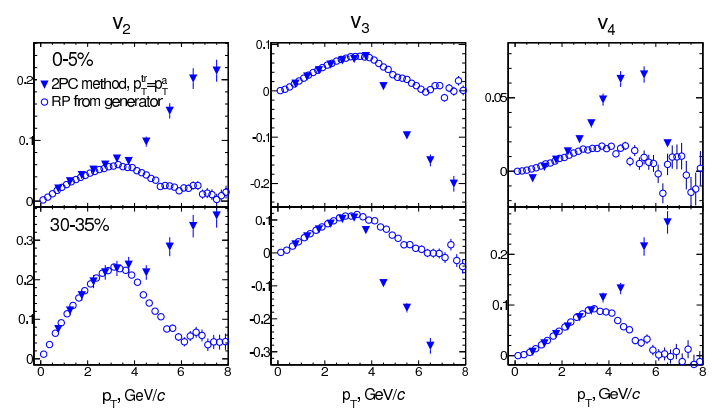

Figure 6: Single-particle flow harmonics $v_{n}$ obtained directly (open circles) and extracted from $V_{n}$ (solid triangles) in $\mathrm{Pb}+\mathrm{Pb}$ collisions at $\sqrt{s}=$ $2.76 \mathrm{TeV}$ with centrality $0-5 \%$ (upper row) and $30-35 \%$ (bottom row).

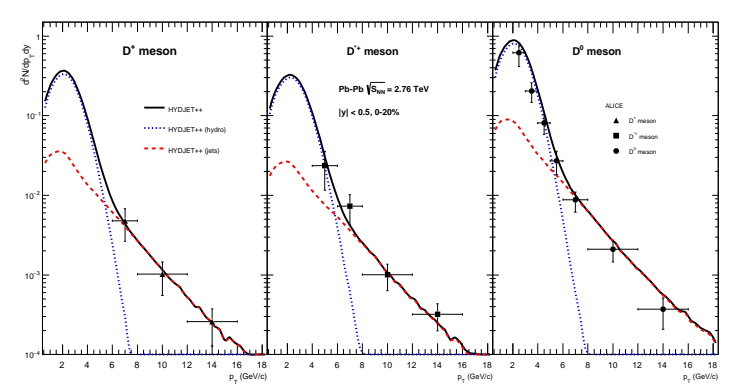

Figure 7: $p_{T}$-spectra of $D^{ \pm}$(left), $D^{* \pm}$ (middle), and $D^{0}$ (right panel) at midrapidity $|y|<0.5$ in $\mathrm{Pb}+\mathrm{Pb}$ collisions at $\sqrt{s}=2.76 \mathrm{TeV}$ with centrality $0-20 \%$. Curves represent hydro (dotted), hard (dashed) and all (solid) processes, symbols denote ALICE data [20].

\section{References}

[1] I.P. Lokhtin, L.V Malinina, S.V. Petrushanko, A.M. Snigirev, I. Arsene, K. Tywoniuk, Comput. Phys. Commun. 180, 779 (2009).

[2] N.S. Amelin et al., Phys. Rev. C 74, 064901 (2006).

[3] N.S. Amelin et al., Phys. Rev. C 77, 014903 (2008).

[4] I.P. Lokhtin, A.M. Snigirev, Eur. Phys. J. C 45, 211 (2006).

[5] G. Eyyubova et al., Phys. Rev. C 80, 064907 (2009).

[6] E.E. Zabrodin et al., J. Phys. G 37, 094060 (2010).

[7] I.P. Lokhtin et al., Eur. Phys. J. C 72, 2045 (2012).

[8] L.V. Bravina et al., Eur. Phys. J. C 74, 2807 (2014).

[9] J. Crkovská et al., Phys. Rev. C 95, 014910 (2017).

[10] E.E Zabrodin et al., J. Phys.: Conf. Ser. 668, 012099 (2016).

[11] L. Bravina, B.H. Brusheim Johansson, G. Eyyubova, E. Zabrodin, Phys. Rev. C 87, 034901 (2013).

[12] L.V. Bravina et al., Phys. Rev. C 89, 024909 (2014).

[13] L.V. Bravina et al., Eur. Phys. J. C 75, 588 (2015).

[14] U. Wiedemann, Phys. Rev. C 57, 266 (1998).

[15] S. Chatrchyan et al. (CMS Collaboration), Phys. Rev. C 87, 014902 (2013).

[16] G. Aad et al. (ATLAS Collaboration), Phys. Rev. C 86, 014907 (2012).

[17] B. Abelev et al., (ALICE Collaboration), Phys. Lett. B 719, 18 (2013).

[18] G.Kh. Eyyubova et al., Phys. Rev. C 91, 064907 (2015).

[19] I.P. Lokhtin et al., J. Exp. Theor. Phys. 124, 244 (2017).

[20] B. Abelev et al., (ALICE Collaboration), JHEP 1209, 112 (2012). 